\title{
Finite entanglement entropy in asymptotically safe quantum gravity
}

\author{
Carlo Pagani and Martin Reuter \\ Institute of Physics, Johannes Gutenberg University Mainz, \\ Staudingerweg 7, D-55099 Mainz, Germany \\ E-mail: capagani@uni-mainz.de, reuter@thep.physik.uni-mainz.de
}

ABSTRACT: Entanglement entropies calculated in the framework of quantum field theory on classical, flat or curved, spacetimes are known to show an intriguing area law in four dimensions, but they are also notorious for their quadratic ultraviolet divergences. In this paper we demonstrate that the analogous entanglement entropies when computed within the Asymptotic Safety approach to background independent quantum gravity are perfectly free from such divergences. We argue that the divergences are an artifact due to the over-idealization of a rigid, classical spacetime geometry which is insensitive to the quantum dynamics.

KeYwords: Models of Quantum Gravity, Renormalization Group

ARXiv EPrint: 1804.02162 


\section{Contents}

1 Introduction 1

2 Entanglement entropy on a rigid background 3

3 Entanglement entropy in QEG $\quad 6$

$\begin{array}{lll}4 & \text { Summary } & 10\end{array}$

\section{Introduction}

One of the most remarkable, and in a way enigmatic, properties of Quantum Mechanics is the occurrence of entangled states and the possibility that local measurements instantaneously affect the result of local measurements far away. While deeply intriguing as a physical phenomenon in its own right, entanglement also received considerable attention from an "applied" perspective, being at the heart of many modern developments in quantum computation and information theory for example. An improved understanding of the entanglement structure of quantum many body systems allowed in particular developing new numerical algorithms which can help in lowering the computational effort of the simulations $[1,2]$.

A frequently used quantity that can quantify the amount of entanglement, at least in pure quantum states, is the entanglement entropy. Let $\rho=|\psi\rangle\langle\psi|$ denote the density operator of an arbitrary quantum system in the pure state $|\psi\rangle$. We assume that the pertinent Hilbert space is a direct product $\mathcal{H}=\mathcal{H}_{A} \otimes \mathcal{H}_{B}$, and that we are only interested in predictions for measurements which affect the subspace $\mathcal{H}_{A}$ alone. Such predictions are encoded in the reduced density matrix $\rho_{A}=\operatorname{Tr}_{B}[\rho]$, where $\operatorname{Tr}_{B}$ denotes the partial trace over $\mathcal{H}_{B}$. Then, by definition, the entanglement entropy related to the $A-B$ decomposition of the total system equals the von Neumann entropy of $\rho_{A}$ :

$$
\mathcal{S}_{A}=-\operatorname{Tr}\left[\rho_{A} \log \rho_{A}\right] .
$$

In practical calculation $\mathcal{S}_{A}$ is often represented as the limit

$$
\mathcal{S}_{A}=-\lim _{n \rightarrow 1} \frac{\partial}{\partial n} \operatorname{Tr}\left[\rho_{A}^{n}\right]
$$

and the replica trick is invoked in order to evaluate $\operatorname{Tr}\left[\rho_{A}^{n}\right]$. The latter consists in calculating $\operatorname{Tr}\left[\rho_{A}^{n}\right]$ for positive integers $n$ and then analytically continue it to a domain in the complex plane. If this step can be justified, calculating $\operatorname{Tr}\left[\rho_{A}^{n}\right]$ can be seen to boil down to the evaluation of a certain partition function; it generalizes the ordinary partition function governing the quantum system considered in that it is defined over a modified, 
more complicated base space, a kind of Riemann surface which may cover the original base space more than once.

Consider for example a free quantum field on Minkowski space, with coordinates $(t, x, y, z)$, and introduce a surface $\Sigma$ by the condition $t=0, x=0$. This surface separates the time slice at $t=0$ in two parts, $x<0$ and $x>0$. If we now define the subsystems $A$ and $B$ as comprised of the field degrees of freedom at $x<0$ and $x>0$, respectively, the ensuing entanglement entropy $\mathcal{S}_{A} \equiv \mathcal{S}$ is given by [3-5]

$$
\mathcal{S}=\left.\left[1+2 \pi \frac{d}{d \delta}\right] \log Z_{\delta}\right|_{\delta=0} .
$$

Here $Z_{\delta}$ is a standard partition function, albeit not on a Minkowski space but on a conical spacetime with a deficit angle $\delta$. Evaluating (1.3), ultraviolet (UV) divergences are encountered, and a short distance cutoff, $\varepsilon$, needs to be introduced, yielding

$$
\mathcal{S}=\frac{\nu}{48 \pi} \frac{A}{\varepsilon^{2}}
$$

In this formula, $\nu$ is a constant which depends on the type of field $(\nu=1$ for a scalar, for example) and $A$ denotes the area of $\Sigma$. Hence, it is meaningful to speak of an entropy per area when $\Sigma$ is infinitely extended. However, $\mathcal{S} / A$ suffers from an UV divergence, it diverges quadratically when the cutoff is removed $(\varepsilon \rightarrow 0)$.

The result (1.4) is valid under more general conditions actually. On any (nondynamical) curved spacetime, and for an arbitrary closed smooth surface $\Sigma$, equation (1.4) gives the leading order contribution to the entanglement entropy.

Clearly, the physical interpretation of (1.4) is hampered by its UV divergence which cannot be "renormalized away" straightforwardly. Nevertheless, ever since its first discovery [6-8], the fact that $\mathcal{S}$ is proportional to the surface area rather than the volume of the subsystem traced over has sparked considerable interest and research activities [3, 6-22]. One of the reasons is clearly the similarity of (1.4) and the Bekenstein-Hawking entropy in black hole thermodynamics,

$$
\mathcal{S}_{\mathrm{BH}}=\frac{A}{4 G}
$$

with $A$ denoting the area of the horizon now [9-11]. This similarity inspired attempts to partially or fully explain $\mathcal{S}_{\mathrm{BH}}$ as an entanglement entropy, and thereby absorb the divergence of $\mathcal{S}$ in a renormalized Newton constant. (We refer to [5] for a comprehensive account.)

The present paper is dedicated to the entanglement entropy (1.4) in its own right, i.e. without reference to black holes or other special systems. Trying to pin down the physical origin of its quadratic divergence, we are going to analyze what happens to the entanglement entropy when the above setting of quantum field theory on classical spacetimes is generalized to full-fledged background independent quantum gravity [23]. Concretely, we shall employ the Asymptotic Safety approach [24, 25] to Quantum Einstein Gravity (QEG) [26-30].

As we are going to argue, the divergence present in the standard result (1.4) originates from the fact that it answers, or tries to answer, an unphysical question that could never 
arise in a real physical experiment. The over-idealization consists in considering "test fields" on an externally prescribed classical spacetime. Instead, if the entanglement is studied in a universe where the geometry is free to adjust itself dynamically according to the gravitational dynamics implied by Asymptotic Safety, the corresponding entropy turns out to be finite.

This is even the more remarkable as a number of quantum gravity models are known to fail in rendering the entropy finite [31] (see however [32] for a proposal achieving a finite entropy in the context of emergent gravity). It should be also emphasized that the proposed non-perturbative mechanism for achieving a finite entanglement entropy does not rely on "hiding" its divergences in Newton's constant or similar couplings which parametrize the action functional.

The rest of this paper is organized as follows. As a preparation we briefly recall in section 2 the derivation of equation (1.4) for classical spacetimes. We also show how it relates in a natural way to the Effective Average Action (EAA), the scale dependent functional that is used in the Asymptotic Safety program. Then, in section 3, we proceed to QEG and analyze the entanglement entropy in a universe with a scale dependent spacetime geometry which is governed by an asymptotically safe renormalization group flow.

\section{Entanglement entropy on a rigid background}

(A) For any free matter field $\Phi$, governed by a quadratic action $S[\Phi]$, the evaluation of the entropy by means of equation (1.3) consists in computing a one-loop determinant on a locally flat spacetime with a conical defect, $\log Z_{\delta}=-\frac{1}{2} \log \operatorname{det}\left(S^{(2)}\right)$. Here $S^{(2)}$ denotes the Hessian operator of $S$. For a scalar, say, $S^{(2)}=-\square+m^{2}$. Standard manipulations lead to the regularized proper time representation

$$
\log Z_{\delta}=\frac{1}{2} \int_{\varepsilon^{2}}^{\infty} \frac{d t}{t} K_{\delta}(t), \text { with } K_{\delta}(t) \equiv \operatorname{Tr}\left[e^{-t S^{(2)}}\right] .
$$

Here the length parameter $\varepsilon$ is introduced as a short distance cutoff in order to cure the divergence of the $t$-integral at the lower limit. So the essential ingredient we need is the heat kernel $K_{\delta}(t)$ as a function of the deficit angle $\delta$,

$$
\mathcal{S}=\frac{1}{2} \lim _{\delta \rightarrow 0} \int_{\varepsilon^{2}}^{\infty} \frac{d t}{t}\left[1+2 \pi \frac{d}{d \delta}\right] K_{\delta}(t)
$$

For a real, massless, minimally coupled scalar, the relevant part of $K_{\delta}(t)$ can be found to be $[33,34]$ :

$$
K(t)=\frac{A}{(4 \pi t)}\left[\frac{\pi L^{2}}{(4 \pi t)}\left(1-\frac{\delta}{2 \pi}\right)+\frac{\delta}{12 \pi}+O\left(\delta^{2}\right)+O\left(\frac{t}{L^{2}}\right)\right]
$$

Here we set $A L^{2} \equiv \int d^{4} x$ for the $4 D$ Euclidean volume. Using (2.3) in (2.2) one obtains exactly the anticipated result for the entanglement entropy, equation (1.4), with $\nu=1$ for the real scalar. Other systems of (higher spin) free fields lead to an analogous formula with other values of the finite constant $\nu$, see [5] for a detailed discussion. 
(B) As a further preparation for the case of quantum gravity let us explain how the above standard calculation should be interpreted within the general framework of the EAA and the functional renormalization group [35].

The EAA for a scalar on a classical spacetime, $\Gamma_{k}[\Phi]$, can be seen as the ordinary effective action for a field whose bare action under the functional integral has been augmented by a mode cutoff term: $S[\Phi] \rightarrow S[\Phi]+\frac{1}{2} \int \Phi \mathcal{R}_{k} \Phi$. The operator $\mathcal{R}_{k} \equiv k^{2} R^{(0)}\left(-\square / k^{2}\right)$ implements an infrared (IR) cutoff by giving a non-zero mass square $\mathcal{R}_{k}=k^{2}$ to the low momentum modes contained in $\Phi$, while annihilating the others, $\mathcal{R}_{k}=0$. This modification leads to the following variant of the partition function on the cone:

$$
\log Z_{\delta}(k)=\frac{1}{2} \int_{\varepsilon^{2}}^{\infty} \frac{d t}{t} K_{\delta}(t), \text { with } K_{\delta}(t) \equiv \operatorname{Tr}\left[e^{-t\left(S^{(2)}+\mathcal{R}_{k}\right)}\right] .
$$

A simple way of analyzing (2.4) is to exploit that the precise form of $\mathcal{R}_{k}$ is largely arbitrary. In any case $\mathcal{R}_{k}$ will leave the contribution to the trace coming from the high momentum modes untouched, while that of the low momentum modes receives an additional factor $\rho(t) \sim e^{-k^{2} t}$, or a qualitatively similar one, which then suppresses the integrand of the $t$-integral at large $t \gtrsim 1 / k^{2}$. Hence, rather than choosing a specific $\mathcal{R}_{k}$ and computing the resulting $\rho(t)$, we may equally well select right away a suitable function $\rho(t)$ with the correct properties, $\rho\left(t \gtrsim 1 / k^{2}\right) \approx 0$ and $\rho\left(t \lesssim 1 / k^{2}\right) \approx 1$. The simplest choice is the step function $\rho(t)=\theta\left(k^{-2}-t\right)$ which, of course, amounts to a version of the Schwinger's proper time regularization [36, 37]. Applied to (2.4) it yields

$$
\log Z_{\delta}(k)=\frac{1}{2} \int_{\varepsilon^{2}}^{k^{-2}} \frac{d t}{t} K_{\delta}(t)
$$

with the same kernel $K_{\delta}(t)$ as in (2.1).

By taking the $k$-derivative of (2.5) we can get rid of the UV cutoff $\varepsilon$ at this point: $k \partial_{k} \log Z_{\delta}(k)=-K_{\delta}\left(k^{-2}\right)$. Associating a scale dependent entropy $\mathcal{S}(k)$ to $Z_{\delta}(k)$ via $(1.3)$, we obtain

$$
k \partial_{k} \mathcal{S}(k)=-\lim _{\delta \rightarrow 0}\left[1+2 \pi \frac{d}{d \delta}\right] K_{\delta}\left(k^{-2}\right)
$$

which evaluates to the following simple RG equation for the, now scale dependent, entanglement entropy:

$$
k \partial_{k} \mathcal{S}(k)=-\frac{\nu}{24 \pi} A[\bar{g}] k^{2} .
$$

Here we wrote $A \equiv A[\bar{g}]$ to emphasize the fact that $A$ is a proper area with respect to a classical, externally prescribed metric, $\bar{g}_{\alpha \beta}$.

By adopting the discussion in [5] it is easy to see that (2.6) holds not only in flat space but also yields the leading scale dependence on curved classical spacetimes with any metric $\bar{g}_{\alpha \beta}$. Furthermore, equation (2.6) is equivalent to the RG equation discussed in [21] which employs a more general cutoff. 
(C) At this point we want to emphasize that in the EAA framework one usually regards the RG equations, requiring no UV cutoff, as having a more fundamental status than the functional integral from which they are derived in a formal way only. ${ }^{1}$ In particular this is the stance taken in the Asymptotic Safety program. This concerns not only the RG equations for the running couplings which parametrize the action functional $\Gamma_{k}$ itself, but also the RG equations for the co-evolving running parameters appearing, for example, in composite operators or observables that do not correspond to terms in $\Gamma_{k}$ [38-40]. In this sense, the entanglement entropy $\mathcal{S}(k)$ is an example of the latter case. Conceptually speaking, it is an "observable" that has a scale dependence in its own right, its RG running depends on the EAA, $\Gamma_{k}$, at least in sufficiently complex truncations.

Like the EAA itself, the co-evolving quantities, too, are defined in the "continuum limit" on the basis of their RG flow. Hence the UV renormalization problem translates into the task of finding complete, i.e. fully extended, solutions (trajectories) to all RG equations, those of the co-evolving quantities included [28].

Let us illustrate this shifted viewpoint by the example of $\mathcal{S}(k)$. While, conceptually speaking, we consider $\mathcal{S}(k)$ a co-evolving quantity with respect to some trajectory of $\Gamma_{k}$, equation (2.6) happens to be simple enough to require no input from $\Gamma_{k}$ to be integrated:

$$
\mathcal{S}\left(k_{2}\right)-\mathcal{S}\left(k_{1}\right)=-\frac{\nu}{48 \pi} A[\bar{g}]\left(k_{2}^{2}-k_{1}^{2}\right)
$$

This difference of two entropies is the contribution of the field modes with momenta in the interval $\left[k_{1}, k_{2}\right]$. We are particularly interested in the limits $k_{1} \rightarrow 0$ and $k_{2} \rightarrow \infty$. The first limit is unproblematic, yielding

$$
\mathcal{S}(0)-\mathcal{S}\left(k_{2}\right)=\frac{\nu}{48 \pi} A[\bar{g}] k_{2}^{2}
$$

Obviously $k_{2}$ plays the role of the UV cutoff here. In the jargon of standard field theory one would refer to $\mathcal{S}\left(k_{2}\right)$ as the "bare", and to $\mathcal{S}(0)$ as the "renormalized" or "physical" quantity. From the EAA perspective, equation (2.8) corresponds to a finite segment of the RG trajectory, $\left\{\Gamma_{k}, k \in\left[0, k_{2}\right]\right\}$, whose lower endpoint $\Gamma_{0}=\Gamma$ equals the standard effective action (with a UV cutoff), having an associated entropy $\mathcal{S}(0)$.

It remains to take the second limit, $k_{2} \rightarrow \infty$, in which $\Gamma_{k \rightarrow \infty}$ is known to approach the classical (bare) action, $S$, essentially. The natural value of the associated entropy is $\mathcal{S}\left(k_{2} \rightarrow \infty\right)=0$ since $\Gamma_{k \rightarrow \infty}$ defines the limiting case of the effective field theory with no quantum fluctuations integrated out yet. Now, ideally, we would let $k_{2} \rightarrow \infty$ in equation (2.8), keeping $\mathcal{S}\left(k_{2}\right)=0$ fixed, and thereby obtain a finite physical value for the entropy, $\mathcal{S}(0)$. But clearly this is thwarted by the $k_{2}^{2}$-dependence on the r.h.s. of $(2.8)$ which causes $\mathcal{S}(0) \propto k_{2}^{2}$ to diverge. In this manner we re-discover the quadratic divergence of the entanglement entropy in the framework of the EAA. It is signalled by the non-existence of an RG trajectory that extends to all $k \in[0, \infty)$.

Next let us see how the situation changes in quantum gravity.

\footnotetext{
${ }^{1}$ That is, in presence of a UV regulator.
} 


\section{Entanglement entropy in QEG}

Up to now we considered matter fields in a prescribed classical background spacetime. Now we go on to Quantum Einstein Gravity (QEG) as defined by a complete, asymptotically free RG trajectory $\Gamma_{k}\left[h_{\alpha \beta}, \Phi ; \bar{g}_{\alpha \beta}\right], k \in[0, \infty)$. As usual, $h_{\alpha \beta}$ and $\bar{g}_{\alpha \beta}$ denote the metric fluctuation and the background metric, respectively. ${ }^{2}$ While our arguments are general, in explicit calculations we will employ the single-metric Einstein-Hilbert truncation coupled to the matter fields $\Phi$; the only running gravitational couplings are the Newton's constant $G(k)$ and the cosmological constant $\Lambda(k)$ then $[25,41]$. It is assumed that the matter fields combined in $\Phi$ are such that they do not destroy the Asymptotic Safety of pure gravity [97].

We may also assume that the reconstruction problem [42] has been solved within the truncation considered. As a result, we have a regularized functional integral at our disposal,

$$
\int \mathcal{D}_{\Lambda} \hat{h} \mathcal{D}_{\Lambda} \hat{\Phi} e^{-S[\hat{h}, \hat{\Phi} ; \bar{g}]}
$$

which approaches a well defined limit when its UV regulator is removed $(\Lambda \rightarrow \infty)$ and which reproduces the asymptotically safe RG trajectory. (See [42] for a detailed discussion.)

(A) What is the meaning of the calculation in section 2 in the Asymptotic Safety context, if any? First of all, as it stands the result for the entanglement entropy refers to a free field. So let us assume that among the matter fields $\Phi$ there is at least one that appears quadratically in the fixed point action, ${ }^{3}$ and let us compute its contribution to the entanglement entropy.

In the gravitational EAA approach, Background Independence is established by studying the dynamics of the metric fluctuation $h_{\alpha \beta}=g_{\alpha \beta}-\bar{g}_{\alpha \beta}$ and matter fields on all backgrounds simultaneously, i.e. $\bar{g}_{\alpha \beta}$ should be left completely arbitrary in the calculation of $\Gamma_{k}$ and the concomitant running quantities.

In this spirit, we now interpret equation (2.6) as the result of a calculation in an arbitrary but fixed, classical background metric, $\bar{g}_{\alpha \beta}$. So at this point $k \partial_{k} \mathcal{S}(k)$ should be understood as a functional of $\bar{g}_{\alpha \beta}$.

(B) Quantum gravity, and specifically QEG, differs most fundamentally from any standard quantum field theory in that it must dynamically generate the spacetime geometry in which all other physics is going to take place then. In particular the theory should be able to distinguish physically realistic, stable states $|\psi\rangle$ from unstable or impossible ones that would never be seen in Nature. The EAA encodes information about physically acceptable states via the metric expectation value it gives rise to, $\left\langle\psi\left|\hat{g}_{\alpha \beta}\right| \psi\right\rangle=g_{\alpha \beta}$.

In the background field formalism, knowing $\Gamma_{k}$, we can search for self-consistent background metrics $\bar{g}_{\alpha \beta}=\left(\bar{g}_{k}^{\mathrm{sc}}\right)_{\alpha \beta}$. By definition, when the $h_{\alpha \beta}$ fluctuations (and the matter fields) are quantized in a self-consistent background, $\hat{h}_{\alpha \beta}$ has vanishing expectation value,

\footnotetext{
${ }^{2}$ We suppress the Faddeev-Popov ghosts here.

${ }^{3}$ Presumably, this is not very restrictive [43, 44].
} 
$\left\langle\hat{h}_{\alpha \beta}\right\rangle \equiv h_{\alpha \beta}=g_{\alpha \beta}-\bar{g}_{\alpha \beta}=0$, and so $g_{\alpha \beta}=\bar{g}_{\alpha \beta}$. In these special backgrounds the quantum fluctuations are particularly tame, and we may regard $g_{\alpha \beta}=\bar{g}_{\alpha \beta}=\left(\bar{g}_{k}^{\mathrm{sc}}\right)_{\alpha \beta}$ as the expectation value of the metric operator in a physically realistic state. ${ }^{4}$

Self-consistent backgrounds are found by solving the tadpole equation [45]:

$$
\left.\frac{\delta}{\delta h_{\alpha \beta}(x)} \Gamma_{k}[h ; \bar{g}]\right|_{h=0, \bar{g}=\bar{g}_{k}^{\mathrm{sc}}}=0 .
$$

Moreover, thanks to the tadpole equation (3.1), the self-consistent background can also be employed to compute the partition function of the system. A detail discussion regarding the properties of such a partition function can be found in [45]. The so computed partition function is then a functional of the self-consistent background: $Z\left[\bar{g}_{k}^{\mathrm{sc}}\right]$.

According to the discussion of section 2, in order to compute the entanglement entropy via the replica trick, one must introduce a deficit angle in the geometry of the system and remove the singularity at the end of the calculation. Namely, one must evaluate the quantity $Z\left[\bar{g}_{k, \delta}^{\mathrm{sc}}\right]$, where $\delta$ is the deficit angle. Note that in general there is no reason for $Z\left[\bar{g}_{k, \delta}^{\mathrm{sc}}\right]$ to be determined directly by $Z\left[\bar{g}_{k}^{\mathrm{sc}}\right]$ since the introduction of the deficit angle changes the topology of the spacetime and a new calculation is required. ${ }^{5}$

Focusing on the Einstein-Hilbert truncation now, the tadpole equation happens to have the same structure as the classical Einstein equation:

$$
R_{\nu}^{\mu}\left(\bar{g}_{k}^{\mathrm{sc}}\right)-\frac{1}{2} \delta_{\nu}^{\mu} R\left(\bar{g}_{k}^{\mathrm{sc}}\right)+\Lambda(k) \delta_{\nu}^{\mu}=0 .
$$

Since under rescalings of the metric the Ricci tensor behaves as $R^{\mu}{ }_{\nu}\left(c^{2} \bar{g}_{k}^{\mathrm{sc}}\right)=c^{-2} R_{\nu}^{\mu}\left(\bar{g}_{k}^{\mathrm{sc}}\right)$, it follows that solutions to (3.2) respond to changes of the cosmological constant in such a way that $\Lambda(k)\left(\bar{g}_{k}^{\mathrm{sc}}\right)_{\alpha \beta}=$ const. It proves convenient to introduce an arbitrary normalization scale $\mu$ in order to write this relation as $\Lambda(k)\left(\bar{g}_{k}^{\mathrm{sc}}\right)_{\alpha \beta}=\Lambda(\mu)\left(\bar{g}_{\mu}^{\mathrm{sc}}\right)_{\alpha \beta}$, or as

$$
\left(\bar{g}_{k}^{\mathrm{sc}}\right)_{\alpha \beta}=\frac{\Lambda(\mu)}{\Lambda(k)}\left(\bar{g}_{\mu}^{\mathrm{sc}}\right)_{\alpha \beta}=\frac{\mu^{2} \lambda(\mu)}{k^{2} \lambda(k)}\left(\bar{g}_{\mu}^{\mathrm{sc}}\right)_{\alpha \beta} .
$$

In the second equality we inserted the dimensionless cosmological constant $\lambda(k)=\Lambda(k) / k^{2}$, and correspondingly for $k=\mu$.

Likewise we redefine the field variables by writing them as dimensionless multiples of the cutoff, or appropriate powers thereof. The dimensionless metric coefficients are then $\tilde{g}_{\alpha \beta} \equiv k^{2} g_{\alpha \beta}$ and $\tilde{\bar{g}}_{\alpha \beta} \equiv k^{2} \bar{g}_{\alpha \beta}$, and so, from (3.3):

$$
\widetilde{\left(\bar{g}_{k}^{\mathrm{sc}}\right)_{\alpha \beta}}=\frac{\lambda(\mu)}{\lambda(k)} \widetilde{\left(\bar{g}_{\mu}^{\mathrm{sc}}\right)_{\alpha \beta}}
$$

\footnotetext{
${ }^{4}$ Note that deciding for a self-consistent background is more special than merely "going on-shell". When split symmetry is broken, the two notions are inequivalent since a non-zero $h_{\alpha \beta}=\left\langle\hat{h}_{\alpha \beta}\right\rangle$ cannot straightforwardly be absorbed into the background metric. Recall also [45] that the general effective field equation for configurations $h_{\alpha \beta} \neq 0$ is more complicated than the tadpole equation (3.1) as it contains an additional term $\propto \mathcal{R}_{k} h_{\alpha \beta}$, which would affect the argument below.

${ }^{5}$ If the EAA was computed keeping track also of the topology dependence, then it may be possible to evaluate the entanglement entropy directly from the EAA itself.
} 
Let us recall that in the case of an asymptotically safe UV limit it is the dimensionless couplings that assume fixed point values. For instance, $\lambda(k)$ approaches a finite number $\lim _{k \rightarrow \infty} \lambda(k)=\lambda_{*}$. Accordingly, it is the dimensionless form of the tadpole equation that continues to be meaningful in the limit of $k \rightarrow \infty$, admitting a finite solution

$$
\widetilde{\left(\bar{g}_{*}^{\mathrm{sc}}\right)_{\alpha \beta}}=\lim _{k \rightarrow \infty} \widetilde{\left(\bar{g}_{k}^{\mathrm{sc}}\right)_{\alpha \beta}}=\frac{1}{\lambda_{*}} \lambda(\mu) \widetilde{\left(\bar{g}_{\mu}^{\mathrm{sc}}\right)_{\alpha \beta}} .
$$

(C) After these preparations we return to the entanglement entropy and reconsider the calculation of section 2 within QEG. In order to obtain the corresponding entropy $\mathcal{S}_{\mathrm{QEG}}(k)$ we choose $\bar{g}_{\alpha \beta}$ in the final result for a rigid background, equation (2.8), to be a self-consistent one for the corresponding scale, $\left(\bar{g}_{k}^{\mathrm{sc}}\right)_{\alpha \beta}$. In this manner we obtain the entanglement entropy related to a system of fields inhabiting a spacetime which is indeed physically realizable, or, at the very least, is much closer to a realizable one than it would be on a generic background. Clearly this is a necessary prerequisite if the entropy computed is to have a physical meaning, and hence a reason to be finite.

Thus we obtain from equation (2.8), writing $k=k_{2}$ from now on,

$$
\begin{aligned}
\mathcal{S}_{\mathrm{QEG}}(0)-\mathcal{S}_{\mathrm{QEG}}(k) & =\frac{\nu}{48 \pi} A\left[\bar{g}_{k}^{\mathrm{sc}}\right] k^{2} \\
& =\frac{\nu}{48 \pi} A\left[k^{2} \bar{g}_{k}^{\mathrm{sc}}\right] .
\end{aligned}
$$

Here we also exploited the fact that the area scales as $A\left[c^{2} g_{\alpha \beta}\right]=c^{2} A\left[g_{\alpha \beta}\right]$. As a result, the entropy difference (3.6) depends on the scale $k$ only via the dimensionless self-consistent metric, that is $\widetilde{\left(\bar{g}_{k}^{\mathrm{sc}}\right)}$ :

$$
\mathcal{S}_{\mathrm{QEG}}(0)-\mathcal{S}_{\mathrm{QEG}}(k)=\frac{\nu}{48 \pi} A\left[\widetilde{\left(\bar{g}_{k}^{\mathrm{sc}}\right)}\right] .
$$

Remarkably enough, when we let $k \rightarrow \infty$ the quantity (3.7) approaches a well defined limit $\mathcal{S}_{\mathrm{QEG}}(0)-\mathcal{S}_{\mathrm{QEG}}(\infty) \equiv \Delta \mathcal{S}_{\mathrm{QEG}}$ :

$$
\Delta \mathcal{S}_{\mathrm{QEG}}=\frac{\nu}{48 \pi} A\left[\widetilde{\left(\widetilde{\bar{g}_{*}^{\mathrm{sc}}}\right)}\right] .
$$

This perfectly finite result for the entanglement entropy in QEG is our main result.

Let us emphasize that the finiteness of the entanglement entropy relies on a dynamical consequence of the effective field equation. In the present case, this manifests itself in the scaling properties of the solution (3.3), and its dimensionless version (3.4), at the fixed point. In the case of the Einstein-Hilbert truncation, this is determined by the growth of the cosmological constant in the UV and the resulting "shrinking" of the metric and all lengths and areas therefore. Let us note that the UV behaviour of the couplings is determined solely by the existence of the fixed point. This entails that our result concerning the "shrinking" of all areas is universal, i.e. it does not depend on a particular RG scheme. In the EAA formalism the above universality can be stated as the fact that the existence of the fixed point does not depend on the form of the chosen cutoff kernel.

Clearly, to define a specific quantum theory a particular trajectory must be selected among those emanating from the fixed point. However, all asymptotically safe trajectories 
lead to the "shrinking" of the metric. Hence, no fine tuning of any couplings is required to remove the quadratic divergence of the entropy.

To match the trajectory against the observed universe, one may fix the trajectory by requiring to recover the observed valued of the cosmological constant in the infrared. This fact has no consequence for the UV behaviour though.

(D) Using (3.5) we may rewrite (3.8) in the more practically applicable forms

$$
\begin{aligned}
\Delta \mathcal{S}_{\mathrm{QEG}} & =\frac{\nu}{48 \pi} \frac{\lambda(\mu)}{\lambda_{*}} \mu^{2} \mathcal{A}(\mu) \\
& =\frac{\nu}{48 \pi} \frac{\Lambda(\mu)}{\lambda_{*}} \mathcal{A}(\mu),
\end{aligned}
$$

where $\mathcal{A}(\mu) \equiv A\left[\bar{g}_{\mu}^{\mathrm{sc}}\right]$ is the dimensionful proper area measured with the background metric at the normalization point $\mu$.

It needs to be emphasized though that the entanglement entropy is independent of the normalization scale $\mu$. In (3.3) we introduced $\mu$ in such a way that the product $\Lambda(\mu)\left(\bar{g}_{\mu}^{\mathrm{sc}}\right)_{\alpha \beta}=\lambda(\mu) \mu^{2}\left(\bar{g}_{\mu}^{\mathrm{sc}}\right)_{\alpha \beta}$ stays constant when $\mu$ is changed, hence $\Lambda(\mu) \mathcal{A}(\mu)=$ $\lambda(\mu) \mu^{2} \mathcal{A}(\mu)$ and therefore the entropy are $\mu$-independent:

$$
\mu \frac{d}{d \mu}\left\{\lambda(\mu) \mu^{2} \mathcal{A}(\mu)\right\}=0
$$

Equation (3.10) may be seen as a simple example of a Callan-Symanzik equation.

(E) Let us consider the following thought experiment to determine the entanglement entropy related to a given surface $\Sigma$. In order to measure the area of $\Sigma$ we must choose a specific "yard stick" (or "microscope"); it is characterized by a certain minimal length which it is able to resolve, $\ell$. The actual measurement consists in using this yard stick to partition $\Sigma$ in little squares of side length $\ell$, and counting the resulting "pixels"; let $\mathcal{N}(\ell)$ denote their total number.

We may assume that the best possible effective field theory description of this measuring procedure is obtained from that EAA which has its scale $k$, or in the present case $\mu$, adapted to the scale of the experiment, $\mu \approx \ell^{-1}$. Hence the measurement is described as taking place in the classical spacetime geometry with $\left.g_{\mu}^{\mathrm{sc}}\right|_{\mu=\ell^{-1}}$. Recall also [46] that the length scale $k^{-1}$ pertaining to $\Gamma_{k}[h ; \bar{g}]$ is a proper length with respect to its second argument, $\bar{g}_{\alpha \beta}$. As a consequence, we can say that the little squares we counted have the proper area $\ell^{2}=\mu^{-2}$ with respect to the optimum self-consistent background metric $g_{\ell^{-1}}^{\mathrm{sc}}$. Hence the result of counting pixels, $\mathcal{N}(\ell)$, has the following interpretation within the effective field theory:

$$
\mathcal{N}(\ell)=\left.\frac{A\left[\bar{g}_{\ell^{-1}}^{\mathrm{sc}}\right]}{\ell^{2}} \equiv \mu^{2} \mathcal{A}(\mu)\right|_{\mu=\ell^{-1}} .
$$

If we accept this interpretation, along with equation (3.9), we can deduce the desired entropy from our pixel count:

$$
\Delta \mathcal{S}_{\mathrm{QEG}}=\frac{\nu}{48 \pi \lambda_{*}} \lambda(1 / \ell) \mathcal{N}(\ell) .
$$


Again, $\mathcal{N}(\ell)$ will not be independent of $\ell$ in general, but the product $\lambda(1 / \ell) \mathcal{N}(\ell)$, and hence the entropy, are $\ell$-independent.

This has an important consequence: if the RG trajectory, and in particular the function $\lambda(k)$ are known, we can determine the entropy on the basis of the formula (3.9) by performing the experiment on any scale we like. This may lead to different numbers of pixels, but the resulting entropy is always the same provided the running of the cosmological constant is taken into account properly.

For example, we could decrease $\ell$ to the point that $\mu=1 / \ell \rightarrow \infty$ enters the scaling regime of the UV fixed point so that $\lambda(1 / \ell) \rightarrow \lambda_{*}$. This limit gives rise to the following representation of the entropy:

$$
\Delta \mathcal{S}_{\mathrm{QEG}}=\frac{\nu}{48 \pi} \mathcal{N}_{*}, \text { where } \mathcal{N}_{*}=\lim _{\ell \rightarrow 0} \mathcal{N}(\ell) .
$$

As soon as the RG trajectory reaches the fixed point regime, $\lambda(\mu)$ stops running. Hence, by (3.10), the area scales as $\mathcal{A}(\mu) \propto 1 / \mu^{2}$ so that $\mathcal{N}(\ell)$ becomes independent of $\ell$; it no longer increases when $\ell$ is decreased even further.

If the EAA follows a type IIIa trajectory [41] which has a long classical regime in the infrared we can use the constant value of the Newton's constant, $G_{\text {class }}$, in order to define Planck units, $\ell_{\mathrm{Pl}} \equiv 1 / m_{\mathrm{Pl}} \equiv \sqrt{G_{\text {class }}}$. Picking $\mu=m_{\mathrm{Pl}}$ leads to a representation of the entanglement entropy that comes close to the Bekenstein-Hawking formula:

$$
\Delta \mathcal{S}_{\mathrm{QEG}}=\frac{\nu}{12 \pi} \frac{\lambda\left(m_{\mathrm{Pl}}\right)}{\lambda_{*}} \frac{\mathcal{A}\left(m_{\mathrm{Pl}}\right)}{4 G_{\mathrm{Pl}}} .
$$

Note however that the prefactor in equation (3.14), while in fact generically of order unity, depends on the matter contents both via $\nu$ and the trajectory, i.e., via the ratio $\lambda\left(m_{\mathrm{Pl}}\right) / \lambda_{*}$.

Finally, let us emphasize that, even if we considered the Einstein-Hilbert truncation for the gravitation EAA, the scaling behaviour of the dimensionless self-consistent metric and of the associated entropy (3.7) is an exact consequence of the Asymptotic Safety scenario as such. It follows that a finite entanglement entropy is achieved also in the case of more refined gravitational EAA truncations. In particular, such extended truncations include higher curvature truncations [47-60], $f(R)$ and infinite dimensional truncations [61-76], bimetric truncations [77-85], truncations for extended theories of gravity [86-90], truncations on foliated spacetimes [91-95], and truncations with different kinds of matter content $[96-115]$. In particular it would be interesting to compute the entanglement entropy for those RG trajectories that are compatible with unitarity $[116,117]$.

\section{Summary}

When defined on a rigid classical spacetime geometry, quantized matter fields are known to give rise to an entanglement entropy which is proportional to the area of the entangling surface, with a factor of proportionality which is quadratically divergent though. In this paper we employed instead a background independent approach to quantum gravity and regarded the entanglement entropy as a scale dependent quantity which RG-evolves in parallel with the Effective Average Action. The latter controls the geometry of spacetime at 
the mean field level, among other things, and in particular it determines the self-consistent background geometries for each scale. The leading term of the entanglement entropy in those geometries turned out to be perfectly finite. The cutoff dependence of the entropy is precisely cancelled by the RG running of the metric in the infinite cutoff limit.

While, for illustrative purposes, we considered the Einstein-Hilbert truncation here, the finiteness of the entropy is a direct consequence of Asymptotic Safety as such and it applies also to more refined truncations schemes.

All that is required is the scaling behaviour of the metric corresponding to a nonGaussian UV fixed point. Hence the finiteness of the leading entropy term is obtained analogously in $d$ spacetime dimensions for surfaces $\Sigma$ of co-dimension two.

From the perspective of Asymptotic Safety, the notorious quadratic UV divergence seems to occur because one is asking an unphysical question, and tries to compute a quantity that never could be measured in Nature, not even in principle. The divergence disappears as soon as we admit that, at asymptotically high scales, spacetime is actually fractal like [118], and carries a metric which strongly depends on the "length of the yard stick" that is used to probe the spacetime.

Open Access. This article is distributed under the terms of the Creative Commons Attribution License (CC-BY 4.0), which permits any use, distribution and reproduction in any medium, provided the original author(s) and source are credited.

\section{References}

[1] S. Haroche and J.-M. Raimond, Exploring the Quantum, Oxford University Press (2006).

[2] I. Bengtsson and K. Życzkowski, Geometry of Quantum States, Cambridge University Press (2006).

[3] C.G. Callan Jr. and F. Wilczek, On geometric entropy, Phys. Lett. B 333 (1994) 55 [hep-th/9401072] [INSPIRE].

[4] D.N. Kabat and M.J. Strassler, A comment on entropy and area, Phys. Lett. B 329 (1994) 46 [hep-th/9401125] [INSPIRE].

[5] S.N. Solodukhin, Entanglement entropy of black holes, Living Rev. Rel. 14 (2011) 8 [arXiv: 1104.3712] [INSPIRE].

[6] R.D. Sorkin, On The Entropy Of The Vacuum Outside A Horizon, in Tenth International Conference on General Relativity and Gravitation, Contributed Papers, vol. II, B. Bertotti, F. de Felice and A. Pascolini eds., Consiglio Nazionale Delle Ricerche (1983).

[7] L. Bombelli, R.K. Koul, J. Lee and R.D. Sorkin, A Quantum Source of Entropy for Black Holes, Phys. Rev. D 34 (1986) 373 [InSPIRE].

[8] M. Srednicki, Entropy and area, Phys. Rev. Lett. 71 (1993) 666 [hep-th/9303048] [INSPIRE].

[9] S.W. Hawking, Particle Creation by Black Holes, Commun. Math. Phys. 43 (1975) 199 [Erratum ibid. 46 (1976) 206] [INSPIRE].

[10] J.D. Bekenstein, Black holes and entropy, Phys. Rev. D 7 (1973) 2333 [INSPIRE]. 
[11] S.W. Hawking, Black Holes and Thermodynamics, Phys. Rev. D 13 (1976) 191 [inSPIRE].

[12] R.M. Wald, The thermodynamics of black holes, Living Rev. Rel. 4 (2001) 6 [gr-qc/9912119] [INSPIRE].

[13] V.P. Frolov and I. Novikov, Dynamical origin of the entropy of a black hole, Phys. Rev. D 48 (1993) 4545 [gr-qc/9309001] [INSPIRE].

[14] L. Susskind and J. Uglum, Black hole entropy in canonical quantum gravity and superstring theory, Phys. Rev. D 50 (1994) 2700 [hep-th/9401070] [InSPIRE].

[15] T. Jacobson, Black hole entropy and induced gravity, gr-qc/9404039 [INSPIRE].

[16] S.N. Solodukhin, The Conical singularity and quantum corrections to entropy of black hole, Phys. Rev. D 51 (1995) 609 [hep-th/9407001] [inSPIRE].

[17] D.V. Fursaev, Black hole thermodynamics and renormalization, Mod. Phys. Lett. A 10 (1995) 649 [hep-th/9408066] [INSPIRE].

[18] J.-G. Demers, R. Lafrance and R.C. Myers, Black hole entropy without brick walls, Phys. Rev. D 52 (1995) 2245 [gr-qc/9503003] [INSPIRE].

[19] D.N. Kabat, Black hole entropy and entropy of entanglement, Nucl. Phys. B 453 (1995) 281 [hep-th/9503016] [INSPIRE].

[20] F. Larsen and F. Wilczek, Renormalization of black hole entropy and of the gravitational coupling constant, Nucl. Phys. B 458 (1996) 249 [hep-th/9506066] [INSPIRE].

[21] T. Jacobson and A. Satz, Black hole entanglement entropy and the renormalization group, Phys. Rev. D 87 (2013) 084047 [arXiv:1212.6824] [INSPIRE].

[22] J.H. Cooperman and M.A. Luty, Renormalization of Entanglement Entropy and the Gravitational Effective Action, JHEP 12 (2014) 045 [arXiv:1302.1878] [INSPIRE].

[23] A. Ashtekar, M. Reuter and C. Rovelli, From General Relativity to Quantum Gravity, arXiv: 1408.4336 [INSPIRE].

[24] S. Weinberg, Ultraviolet Divergences In Quantum Theories Of Gravitation, in General Relativity, an Einstein Centenary Survey, S.W. Hawking and W. Israel eds., Cambridge University Press (1980), pg. 790 [InSPIRE].

[25] M. Reuter, Nonperturbative evolution equation for quantum gravity, Phys. Rev. D 57 (1998) 971 [hep-th/9605030] [INSPIRE].

[26] M. Niedermaier and M. Reuter, The Asymptotic Safety Scenario in Quantum Gravity, Living Rev. Rel. 9 (2006) 5 [INSPIRE].

[27] R. Percacci, An Introduction To Covariant Quantum Gravity And Asymptotic Safety, World Scientific (2017).

[28] M. Reuter and F. Saueressig, Quantum Gravity and the Functional Renormalization Group - The road towards Asymptotic Safety, Cambridge University Press, in press.

[29] M. Reuter and F. Saueressig, Quantum Einstein Gravity, New J. Phys. 14 (2012) 055022 [arXiv: 1202.2274] [INSPIRE].

[30] M. Reuter and F. Saueressig, Asymptotic Safety, Fractals and Cosmology, Lect. Notes Phys. 863 (2013) 185 [arXiv:1205.5431] [INSPIRE].

[31] M. Arzano and G. Calcagni, Finite entanglement entropy and spectral dimension in quantum gravity, Eur. Phys. J. C 77 (2017) 835 [arXiv:1704.01141] [INSPIRE]. 
[32] T. Padmanabhan, Finite entanglement entropy from the zero-point-area of spacetime, Phys. Rev. D 82 (2010) 124025 [arXiv: 1007.5066] [INSPIRE].

[33] J.S. Dowker, Quantum Field Theory on a Cone, J. Phys. A 10 (1977) 115 [InSPIRE].

[34] D.V. Fursaev, Spectral geometry and one loop divergences on manifolds with conical singularities, Phys. Lett. B 334 (1994) 53 [hep-th/9405143] [INSPIRE].

[35] C. Wetterich, Exact evolution equation for the effective potential, Phys. Lett. B 301 (1993) 90 [arXiv:1710.05815] [INSPIRE].

[36] J.S. Schwinger, On gauge invariance and vacuum polarization, Phys. Rev. 82 (1951) 664 [INSPIRE].

[37] W. Dittrich and M. Reuter, Effective Lagrangians In Quantum Electrodynamics, Lect. Notes Phys. 220 (1985) 1 [INSPIRE].

[38] C. Pagani and M. Reuter, Composite Operators in Asymptotic Safety, Phys. Rev. D 95 (2017) 066002 [arXiv:1611.06522] [InSPIRE].

[39] C. Pagani, Note on scaling arguments in the effective average action formalism, Phys. Rev. D 94 (2016) 045001 [arXiv: 1603.07250] [INSPIRE].

[40] C. Pagani and H. Sonoda, Products of composite operators in the exact renormalization group formalism, PTEP 2018 (2018) 023B02 [arXiv:1707.09138] [INSPIRE].

[41] M. Reuter and F. Saueressig, Renormalization group flow of quantum gravity in the Einstein-Hilbert truncation, Phys. Rev. D 65 (2002) 065016 [hep-th/0110054] [INSPIRE].

[42] E. Manrique and M. Reuter, Bare Action and Regularized Functional Integral of Asymptotically Safe Quantum Gravity, Phys. Rev. D 79 (2009) 025008 [arXiv:0811.3888] [INSPIRE].

[43] R. Percacci and G.P. Vacca, Search of scaling solutions in scalar-tensor gravity, Eur. Phys. J. C 75 (2015) 188 [arXiv: 1501.00888] [INSPIRE].

[44] N. Christiansen, D.F. Litim, J.M. Pawlowski and M. Reichert, Asymptotic safety of gravity with matter, Phys. Rev. D 97 (2018) 106012 [arXiv:1710.04669] [INSPIRE].

[45] D. Becker and M. Reuter, Towards a $C$-function in $4 D$ quantum gravity, JHEP 03 (2015) 065 [arXiv: 1412.0468] [INSPIRE].

[46] M. Reuter and J.-M. Schwindt, A Minimal length from the cutoff modes in asymptotically safe quantum gravity, JHEP 01 (2006) 070 [hep-th/0511021] [INSPIRE].

[47] O. Lauscher and M. Reuter, Flow equation of quantum Einstein gravity in a higher derivative truncation, Phys. Rev. D 66 (2002) 025026 [hep-th/0205062] [INSPIRE].

[48] M. Reuter and F. Saueressig, A Class of nonlocal truncations in quantum Einstein gravity and its renormalization group behavior, Phys. Rev. D 66 (2002) 125001 [hep-th/0206145] [INSPIRE].

[49] A. Codello, R. Percacci and C. Rahmede, Ultraviolet properties of $f(R)$-gravity, Int. J. Mod. Phys. A 23 (2008) 143 [arXiv:0705.1769] [InSPIRE].

[50] D. Benedetti, P.F. Machado and F. Saueressig, Asymptotic safety in higher-derivative gravity, Mod. Phys. Lett. A 24 (2009) 2233 [arXiv:0901.2984] [INSPIRE].

[51] D. Benedetti, K. Groh, P.F. Machado and F. Saueressig, The Universal RG Machine, JHEP 06 (2011) 079 [arXiv: 1012.3081] [inSPIRE]. 
[52] S. Rechenberger and F. Saueressig, The $R^{2}$ phase-diagram of $Q E G$ and its spectral dimension, Phys. Rev. D 86 (2012) 024018 [arXiv: 1206.0657] [INSPIRE].

[53] N. Ohta and R. Percacci, Higher Derivative Gravity and Asymptotic Safety in Diverse Dimensions, Class. Quant. Grav. 31 (2014) 015024 [arXiv:1308.3398] [InSPIRE].

[54] D. Benedetti, On the number of relevant operators in asymptotically safe gravity, EPL 102 (2013) 20007 [arXiv: 1301 .4422] [INSPIRE].

[55] K. Falls, D.F. Litim, K. Nikolakopoulos and C. Rahmede, Further evidence for asymptotic safety of quantum gravity, Phys. Rev. D 93 (2016) 104022 [arXiv:1410.4815] [InSPIRE].

[56] A. Eichhorn, The Renormalization Group flow of unimodular $f(R)$ gravity, JHEP 04 (2015) 096 [arXiv: 1501. 05848] [INSPIRE].

[57] N. Ohta, R. Percacci and G.P. Vacca, Flow equation for $f(R)$ gravity and some of its exact solutions, Phys. Rev. D 92 (2015) 061501 [arXiv: 1507.00968] [INSPIRE].

[58] K. Falls, D.F. Litim, K. Nikolakopoulos and C. Rahmede, On de Sitter solutions in asymptotically safe $f(R)$ theories, Class. Quant. Grav. 35 (2018) 135006 [arXiv: 1607.04962] [INSPIRE].

[59] K. Falls and N. Ohta, Renormalization Group Equation for $f(R)$ gravity on hyperbolic spaces, Phys. Rev. D 94 (2016) 084005 [arXiv: 1607.08460] [INSPIRE].

[60] H. Gies, B. Knorr, S. Lippoldt and F. Saueressig, Gravitational Two-Loop Counterterm Is Asymptotically Safe, Phys. Rev. Lett. 116 (2016) 211302 [arXiv: 1601.01800] [INSPIRE].

[61] M. Reuter and H. Weyer, Conformal sector of Quantum Einstein Gravity in the local potential approximation: Non-Gaussian fixed point and a phase of unbroken diffeomorphism invariance, Phys. Rev. D 80 (2009) 025001 [arXiv:0804.1475] [INSPIRE].

[62] D. Benedetti and F. Caravelli, The Local potential approximation in quantum gravity, JHEP 06 (2012) 017 [Erratum ibid. 10 (2012) 157] [arXiv: 1204.3541] [INSPIRE].

[63] M. Demmel, F. Saueressig and O. Zanusso, Fixed-Functionals of three-dimensional Quantum Einstein Gravity, JHEP 11 (2012) 131 [arXiv:1208.2038] [INSPIRE].

[64] J.A. Dietz and T.R. Morris, Asymptotic safety in the $f(R)$ approximation, JHEP 01 (2013) 108 [arXiv: 1211.0955] [INSPIRE].

[65] I.H. Bridle, J.A. Dietz and T.R. Morris, The local potential approximation in the background field formalism, JHEP 03 (2014) 093 [arXiv:1312.2846] [INSPIRE].

[66] J.A. Dietz and T.R. Morris, Redundant operators in the exact renormalisation group and in the $f(R)$ approximation to asymptotic safety, JHEP 07 (2013) 064 [arXiv:1306.1223] [INSPIRE].

[67] M. Demmel, F. Saueressig and O. Zanusso, $R G$ flows of Quantum Einstein Gravity on maximally symmetric spaces, JHEP 06 (2014) 026 [arXiv: 1401.5495] [INSPIRE].

[68] M. Demmel, F. Saueressig and O. Zanusso, $R G$ flows of Quantum Einstein Gravity in the linear-geometric approximation, Annals Phys. 359 (2015) 141 [arXiv:1412.7207] [INSPIRE].

[69] M. Demmel, F. Saueressig and O. Zanusso, A proper fixed functional for four-dimensional Quantum Einstein Gravity, JHEP 08 (2015) 113 [arXiv: 1504.07656] [INSPIRE]. 
[70] N. Ohta, R. Percacci and G.P. Vacca, Renormalization Group Equation and scaling solutions for $f(R)$ gravity in exponential parametrization, Eur. Phys. J. C 76 (2016) 46 [arXiv: 1511.09393] [INSPIRE].

[71] P. Labus, T.R. Morris and Z.H. Slade, Background independence in a background dependent renormalization group, Phys. Rev. D 94 (2016) 024007 [arXiv: 1603.04772] [INSPIRE].

[72] J.A. Dietz, T.R. Morris and Z.H. Slade, Fixed point structure of the conformal factor field in quantum gravity, Phys. Rev. D 94 (2016) 124014 [arXiv:1605.07636] [INSPIRE].

[73] B. Knorr, Infinite order quantum-gravitational correlations, Class. Quant. Grav. 35 (2018) 115005 [arXiv:1710.07055] [INSPIRE].

[74] N. Christiansen, K. Falls, J.M. Pawlowski and M. Reichert, Curvature dependence of quantum gravity, Phys. Rev. D 97 (2018) 046007 [arXiv:1711.09259] [INSPIRE].

[75] K. Falls, C.R. King, D.F. Litim, K. Nikolakopoulos and C. Rahmede, Asymptotic safety of quantum gravity beyond Ricci scalars, Phys. Rev. D 97 (2018) 086006 [arXiv:1801.00162] [INSPIRE].

[76] N. Alkofer and F. Saueressig, Asymptotically safe $f(R)$-gravity coupled to matter I: the polynomial case, arXiv:1802.00498 [INSPIRE].

[77] E. Manrique and M. Reuter, Bimetric Truncations for Quantum Einstein Gravity and Asymptotic Safety, Annals Phys. 325 (2010) 785 [arXiv:0907.2617] [INSPIRE].

[78] E. Manrique, M. Reuter and F. Saueressig, Matter Induced Bimetric Actions for Gravity, Annals Phys. 326 (2011) 440 [arXiv:1003.5129] [INSPIRE].

[79] E. Manrique, M. Reuter and F. Saueressig, Bimetric Renormalization Group Flows in Quantum Einstein Gravity, Annals Phys. 326 (2011) 463 [arXiv: 1006.0099] [INSPIRE].

[80] N. Christiansen, D.F. Litim, J.M. Pawlowski and A. Rodigast, Fixed points and infrared completion of quantum gravity, Phys. Lett. B 728 (2014) 114 [arXiv:1209.4038] [InSPIRE].

[81] A. Codello, G. D'Odorico and C. Pagani, Consistent closure of renormalization group flow equations in quantum gravity, Phys. Rev. D 89 (2014) 081701 [arXiv:1304.4777] [INSPIRE].

[82] N. Christiansen, B. Knorr, J.M. Pawlowski and A. Rodigast, Global Flows in Quantum Gravity, Phys. Rev. D 93 (2016) 044036 [arXiv: 1403.1232] [inSPIRE].

[83] D. Becker and M. Reuter, En route to Background Independence: Broken split-symmetry and how to restore it with bi-metric average actions, Annals Phys. 350 (2014) 225 [arXiv: 1404.4537] [INSPIRE].

[84] N. Christiansen, B. Knorr, J. Meibohm, J.M. Pawlowski and M. Reichert, Local Quantum Gravity, Phys. Rev. D 92 (2015) 121501 [arXiv:1506.07016] [INSPIRE].

[85] B. Knorr and S. Lippoldt, Correlation functions on a curved background, Phys. Rev. D 96 (2017) 065020 [arXiv:1707.01397] [InSPIRE].

[86] J.E. Daum and M. Reuter, Renormalization Group Flow of the Holst Action, Phys. Lett. B 710 (2012) 215 [arXiv:1012.4280] [INSPIRE].

[87] J.E. Daum and M. Reuter, Einstein-Cartan gravity, Asymptotic Safety and the running Immirzi parameter, Annals Phys. 334 (2013) 351 [arXiv:1301.5135] [INSPIRE].

[88] C. Pagani and R. Percacci, Quantization and fixed points of non-integrable Weyl theory, Class. Quant. Grav. 31 (2014) 115005 [arXiv:1312.7767] [INSPIRE]. 
[89] C. Pagani and R. Percacci, Quantum gravity with torsion and non-metricity, Class. Quant. Grav. 32 (2015) 195019 [arXiv: 1506. 02882] [INSPIRE].

[90] M. Reuter and G.M. Schollmeyer, The metric on field space, functional renormalization and metric-torsion quantum gravity, Annals Phys. 367 (2016) 125 [arXiv:1509.05041] [INSPIRE].

[91] E. Manrique, S. Rechenberger and F. Saueressig, Asymptotically Safe Lorentzian Gravity, Phys. Rev. Lett. 106 (2011) 251302 [arXiv:1102.5012] [INSPIRE].

[92] S. Rechenberger and F. Saueressig, A functional renormalization group equation for foliated spacetimes, JHEP 03 (2013) 010 [arXiv:1212.5114] [INSPIRE].

[93] J. Biemans, A. Platania and F. Saueressig, Quantum gravity on foliated spacetimes: Asymptotically safe and sound, Phys. Rev. D 95 (2017) 086013 [arXiv:1609.04813] [INSPIRE].

[94] J. Biemans, A. Platania and F. Saueressig, Renormalization group fixed points of foliated gravity-matter systems, JHEP 05 (2017) 093 [arXiv: 1702.06539] [INSPIRE].

[95] W.B. Houthoff, A. Kurov and F. Saueressig, Impact of topology in foliated Quantum Einstein Gravity, Eur. Phys. J. C 77 (2017) 491 [arXiv:1705.01848] [INSPIRE].

[96] D. Dou and R. Percacci, The running gravitational couplings, Class. Quant. Grav. 15 (1998) 3449 [hep-th/9707239] [inSPIRE].

[97] R. Percacci and D. Perini, Constraints on matter from asymptotic safety, Phys. Rev. D 67 (2003) 081503 [hep-th/0207033] [INSPIRE].

[98] G. Narain and R. Percacci, Renormalization Group Flow in Scalar-Tensor Theories. I, Class. Quant. Grav. 27 (2010) 075001 [arXiv:0911.0386] [INSPIRE].

[99] J.E. Daum, U. Harst and M. Reuter, Non-perturbative QEG Corrections to the Yang-Mills $\beta$-function, Gen. Relativ. Gravit. (2010) [arXiv:1005.1488] [INSPIRE].

[100] S. Folkerts, D.F. Litim and J.M. Pawlowski, Asymptotic freedom of Yang-Mills theory with gravity, Phys. Lett. B 709 (2012) 234 [arXiv:1101.5552] [INSPIRE].

[101] U. Harst and M. Reuter, QED coupled to QEG, JHEP 05 (2011) 119 [arXiv:1101.6007] [INSPIRE].

[102] A. Eichhorn and H. Gies, Light fermions in quantum gravity, New J. Phys. 13 (2011) 125012 [arXiv:1104.5366] [InSPIRE].

[103] A. Eichhorn, Quantum-gravity-induced matter self-interactions in the asymptotic-safety scenario, Phys. Rev. D 86 (2012) 105021 [arXiv:1204.0965] [INSPIRE].

[104] P. Donà and R. Percacci, Functional renormalization with fermions and tetrads, Phys. Rev. D 87 (2013) 045002 [arXiv:1209.3649] [INSPIRE].

[105] P. Donà, A. Eichhorn and R. Percacci, Matter matters in asymptotically safe quantum gravity, Phys. Rev. D 89 (2014) 084035 [arXiv:1311.2898] [INSPIRE].

[106] P. Labus, R. Percacci and G.P. Vacca, Asymptotic safety in $O(N)$ scalar models coupled to gravity, Phys. Lett. B 753 (2016) 274 [arXiv: 1505.05393] [INSPIRE].

[107] K.-y. Oda and M. Yamada, Non-minimal coupling in Higgs-Yukawa model with asymptotically safe gravity, Class. Quant. Grav. 33 (2016) 125011 [arXiv:1510.03734] [INSPIRE]. 
[108] J. Meibohm, J.M. Pawlowski and M. Reichert, Asymptotic safety of gravity-matter systems, Phys. Rev. D 93 (2016) 084035 [arXiv:1510.07018] [InSPIRE].

[109] P. Donà, A. Eichhorn, P. Labus and R. Percacci, Asymptotic safety in an interacting system of gravity and scalar matter, Phys. Rev. D 93 (2016) 044049 [Erratum ibid. D 93 (2016) 129904] [arXiv: 1512.01589] [INSPIRE].

[110] J. Meibohm and J.M. Pawlowski, Chiral fermions in asymptotically safe quantum gravity, Eur. Phys. J. C 76 (2016) 285 [arXiv: 1601.04597] [InSPIRE].

[111] A. Eichhorn, A. Held and J.M. Pawlowski, Quantum-gravity effects on a Higgs-Yukawa model, Phys. Rev. D 94 (2016) 104027 [arXiv: 1604.02041] [InSPIRE].

[112] A. Eichhorn and S. Lippoldt, Quantum gravity and Standard-Model-like fermions, Phys. Lett. B 767 (2017) 142 [arXiv:1611.05878] [INSPIRE].

[113] N. Christiansen and A. Eichhorn, An asymptotically safe solution to the U(1) triviality problem, Phys. Lett. B 770 (2017) 154 [arXiv:1702.07724] [INSPIRE].

[114] A. Eichhorn and A. Held, Top mass from asymptotic safety, Phys. Lett. B 777 (2018) 217 [arXiv: 1707.01107] [INSPIRE].

[115] A. Eichhorn and F. Versteegen, Upper bound on the Abelian gauge coupling from asymptotic safety, JHEP 01 (2018) 030 [arXiv:1709.07252] [INSPIRE].

[116] D. Becker, C. Ripken and F. Saueressig, On avoiding Ostrogradski instabilities within Asymptotic Safety, JHEP 12 (2017) 121 [arXiv:1709.09098] [INSPIRE].

[117] F. Arici, D. Becker, C. Ripken, F. Saueressig and W.D. van Suijlekom, Reflection positivity in higher derivative scalar theories, arXiv:1712.04308 [INSPIRE].

[118] O. Lauscher and M. Reuter, Fractal spacetime structure in asymptotically safe gravity, JHEP 10 (2005) 050 [hep-th/0508202] [INSPIRE]. 